\title{
25 Research Square \\ Developing the First Iranian Guideline for Management of Poor Prognosis Infants
}

\section{Fatemeh Sadat Nayeri}

Tehran University of Medical Sciences

Fariba Asghari

Tehran University of Medical Sciences

Mahmoud Motevasel Arani

Tehran University of Medical Sciences

Mohsen Vigeh

Juntendo University

Maryam Kheyri

Ministry of Health and Medical Education

Ehsan Shamsi-Gooshki

Tehran University of Medical Sciences

Alireza Parsapoor

Tehran University of Medical Sciences

Mamak Shariat ( $\square$ mshariat@tums.ac.ir)

Tehran University of Medical Sciences

Nahid Farrokhzad

Tehran University of Medical Sciences

\section{Research Article}

Keywords: Poor Prognosis Infants, Guideline, Extremely Low Birth Weight, Asphyxia, Prematurity, Decision-making

Posted Date: November 8th, 2021

DOI: https://doi.org/10.21203/rs.3.rs-1008987/v1

License: (c) (1) This work is licensed under a Creative Commons Attribution 4.0 International License. Read Full License 


\section{Abstract}

Background: Significantly improvements in the medical science and advanced in medical equipment and hospital facilities have increased the survival rate of poor prognosis infants (PPIs), including premature/extremely low birth weight, asphyxiated, and born with potentially lethal congenital disorders. However, availability of medical resources was not at the same levels across Iran's cities. Thus, the country health care system encounters to a challenging question about providing advanced care to PPIs.

Methods: The present study aimed to develop a national guideline for management of PPIs. We reviewed 120 articles, protocols, and guidelines, and held 15 sessions with 42 active participants. Semi-structured interviews were performed separately with medical specialist teams, medical ethicists, religious masters (Shia clerics), and parents of PPIs.

Results \& Conclusions: This guideline is the first nationwide attempt to prepare a comprehensive framework that can support prenatal counseling in Iranian hospitals with various medical facilities to achieve more homogeneity in newborn medical care. In addition, we collected an extensive list of lethal congenital malformations, genetic disorders, inborn errors of metabolism and covers the main areas of concern with a clearly states of responsibilities for decision-making regarding prenatal counseling for PPIs across Iran.

\section{Introduction}

Poor prognosis infants (PPI) usually indicates three adverse conditions: prematurity/ extremely low birth weight (ELBW), asphyxia, and born with potentially lethal congenital disorders. Among these, prematurity is one of the most common causes of neonatal death and remains an important public health problem. An estimated one percent of babies have a birth weight of 1,500 grams or lower, of whom more than half will die. In addition, according to the World Health Organization (WHO), about 30 million low birth weight babies are born in the world every year, of whom 15 million are premature [1]. On the other hand, lethal congenital disorders, including genetic disorders, inborn errors of metabolism (IEM), and congenital malformations are other causes of PPI, for many of which there is no effective treatment. Sever asphyxia is an important cause of neonatal morbidity (i.e., brain injury, cerebral palsy, and seizure disorders in later life), and up to $75 \%$ of the survived infants suffer from severe disabilities. The mortality rate is also high in infants with severe asphyxia [2]. In the developed countries, about $10 \%$ of newborns have different levels of asphyxia, of whom 0.1-0.2\% develop severe hypoxic-ischemic encephalopathy (HIE). However, there is not sufficient information about the incidence in developing countries, but it might be higher than the developed countries [3].

Significant improvements in the medical science and advanced in medical equipment and hospital facilities, such as Neonatal Intensive Care Units (NICUs) have increased the survival rate of high-risk neonates. Recent studies have confirmed improvements in the survival rate of poor prognosis infants, including those with extremely preterm birth (22-25 completed weeks), severe asphyxia, multiple 
congenital anomalies, severe IEM, and severe genetic disorders. However, these newborns often have many medical complications and morbidities, which demand higher health system capacities and financial support and apply more emotional pressure to their families. As a result, physicians and families face new challenges regarding decision making about the care of infants with such complicated health conditions. On the other hand, despite all medical efforts, many of such infants die soon after birth or suffer from serious disabilities with frequent re-admissions and/or long-term hospitalizations and a low quality of life. Due to insufficient NICU beds in many countries including Iran, admission of these infants reduces the availability of medical care to some neonates with higher chance of survival [4].

Limit of viability (LoV) is defined a gestational age at which a premature infant has a $50 \%$ chance of long-term survival without major neurological damage [5]. Currently, in the United States, the LoV is a gestational age of 23 to 24 weeks and/or birth weight of $\geq 500 \mathrm{~g}$ [6-8]. The incidence and survival rate of these infants may vary among countries [9]. For example, a study reported a mortality rate of $40 \%$ for ELBW neonates in Iran [10]. On the other word, the survival rate of neonates delivered at higher gestational ages (28 weeks) may be significantly higher (77-82\%) [9].

In many countries, consultation with parents is often carried out before the delivery of poor prognosis babies to provide adequate information about the potential risks and related adverse health consequences. Decision regarding giving intensive treatment to extremely preterm infant is usually made upon consultation with the health care team, social workers, and medical ethicists with the priority of parents' desire [11, 12].

In Iran, hospital facilities, health care providers, and other medical resources are not similar across the country. Thus, the health care system encounters to a challenging question about providing advanced (expensive) care to very poor prognosis neonates. Like many countries, religious believes and medical ethics have important roles in decision-making in such cases in Iran. As Islamic instructions are in favor of protecting the humans' health and life, treatment cannot be waived unless the practitioner is certain of the patient's imminent death. However, the medical support for poor prognosis babies requires many facilities and expert personnel, when is not sufficiently available in all parts of Iran.

Some countries have their own guidelines and recommendations regarding the management of extremely preterm birth, such as "no resuscitation for infants born at 22-24 weeks' gestation" [13] or "give comfort care for infants with a gestational age of 24 weeks, and intensive care becomes a valid option in borderline viable infants with a gestational age $>24$ weeks" [14]. Policymaking and decision frameworks regarding life-sustaining treatment for poor prognosis babies are essential for our country as well. This study aimed to present a national Iranian guideline for decision making regarding the management of poor prognosis newborns which is prepared after consultation with a rage of related scholars and stakeholders neonatologists, obstetricians/ gynecologists, forensic medicine specialists, medical ethicists, religious leaders (Shia clerics), nurses, and parents.

\section{Methods}


Development of the national guideline included two main phases. In the first phase one background research project was done in Tehran University of Medical Sciences (TUMS) by an interdisciplinary research team, headed by an experienced neonatologist. The result of this study was a draft guideline introduced to Iran Ministry of Health and Medical Education (MHME). In the second phase, the proposed guideline was discussed in several official committees and meetings in and was revised into its final form. The first phase, background study, was done in three steps: 1) A metal-analysis study about PPI outcomes in Iran, the Middle East, and Western Asia published from 2000 to 2016, 2) searching the literature, standards, strategies, and clinical guidelines about management of PPIs, 3) A qualitative study using content analysis approach. At first, using selected keywords (Preterm OR very preterm OR premature OR very premature OR low birth weight OR very low birth weight immature) AND (Infants OR newborns OR neonates) AND (mortality OR survival OR morbidity OR outcome OR follow up) in combination with EMRO countries, it was tried to determine the survival rate and long-term complications of PPIs in Iran, Middle East, and West Asian countries. A meta-analysis was published based on the collected data [15]. The online databases that were searched included PubMed, Google Scholar, Web of Sciences, EMBASE, Up to Date, and Scopus. Then, an inferential analysis was performed about PPI management standards, strategies, instructions, and clinical guidelines in other countries using online databases. In the next step, the research team gathered these findings to design a qualitative study using "content analysis" approach.

This qualitative study included fifteen discussion panels as focus group discussions. Semi-structured interviews were performed separately with medical specialist teams and religious masters. A number of medical specialists/ sub-specialists were invited, including neonatologists and pediatric surgeons/ neurologists/ nephrologists/ cardiologists $(n=27)$, obstetricians/ gynecologists/ perinatologists $(n=18)$, forensic medicine specialists $(n=8)$, medical ethicists $(n=14)$, NICU nurses with 10-15 years of work experience $(n=15)$, religious leaders (Shia clerics) $(n=6)$, and parents with PPI at the research hospitals $(n=10)$. The participants were informed about the study purposes and provided their written consent before participation in the study. In addition, an interview guide was prepared in advance to ensure that all of the important areas were covered. In each discussion panel, the participants detailed their experience with management of PPIs and their families. The nurses, practitioners, and medical specialists were interviewed at the university hospitals, parents were interviewed at an appropriate place near the NICU, and religious leaders were interviewed at their offices. The interviews were audio recorded and re-checked with memos. For qualitative analysis, the findings were coded and categorized, and the findings will be published in the near future.

Due to differences in hospitals facilities and health conditions in Iran, the study participants, such as neonatologists and obstetricians/gynecologists (perinatologists) were selected from different parts of the country, mostly from the faculty members of medical universities.

A list of severe life-threatening genetic disorders, hereditary metabolic diseases, and congenital anomalies was also prepared according to the current medical knowledge and experts' experiences (Appendix). For each item, discussion continued until all the members accepted the final list. The findings 
of the current survey were summarized to prepare a uniform guideline, which can be used in all hospital's settings in all cities of Iran.

The initial draft of the guideline was revised by neonatologists, perinatologists and ethicists. They added a glossary, including concepts and definitions of the main conditions in PPIs (Table 1). Then, based on the researchers' opinions and clinical recommendations, the PPIs were divided into four groups as below 25 weeks gestational age, 25-32 weeks gestational age, severe asphyxia, and infants with severe lifethreatening congenital abnormalities.

In phase 2, The resulted guideline was presented to National Clinical Ethics Committee (NCEC), which is the official body embedded inside MHME. At the same time the draft was discussed in Iran Academy of Medical Sciences Then, the draft was sent to the Institute of Theology of Jurisprudential Rulings[1] to receive Islamic leaders' opinions about the guideline content. At the end, the final version of the guideline was accepted in five expert panel discussions including several medical experts (ethics specialists and neonatologists) and the protocol writing members of the Iranian MHME.

After several meetings and long discussions and opinion exchange, the updated version was accepted by the NCEC.

The final version of the guideline was approved by Supreme Council of Medical Ethics, which is the highest-level interdisciplinary and inter-institutional body in Iran health system for ethical issues in health care system.

All the study was carried out in accordance with national codes of ethics for research on human subjects which are compatible with international research ethics standards included in the Declaration of Helsinki. The study protocol was ethically approved by the Research Ethics Committee, Tehran University of Medical Sciences (IR.TUMS.VCR.REC.1397.658) and the survey has conducted under the supervision of Maternal, Neonatal, and Fetal Research Center, TUMS, Tehran, Iran. The study's researchers had sent an invitation letter to all participants for attending to the discussion panels (respond rate of $85 \%$ ) and interviews (respond rate of $70 \%$ ). Prior to volunteers' recruitment, a complete verbal explanation had been given about purpose and procedures of the study, in purely voluntary situation.

Table 1 The keywords definition in the current study 
Severe lifethreatening malformations, genetic and metabolic diseases

Severe asphyxia

Aggressive care

Conservative/ supportive care
Infant who was born with one or more malformations and genetic or metabolic abnormalities, were diagnosed before birth, and died immediately or within a week despite advanced medical and/or surgical procedures

Stage III of hypoxic ischemic encephalopathy (HIE), multiple organ failure following asphyxia, Apgar score $<3$ at 20 minutes with seizures due to asphyxia, or asphyxia with grade IV intraventricular hemorrhage

Need for all clinical procedures preserve the life of the infant, including surgery, cardiopulmonary resuscitation, endotracheal intubation and mechanical ventilation, chest tube insertion, and total parenteral nutrition

Administration of oxygen through non-invasive procedures (hood, nasal cannula, and nasal continuous positive airway pressure or NCPAP), feeding (gastric tube, intravenous line through, and an umbilical artery catheter), nasopharyngeal suction, percutaneous intracardiac catheter, antibiotic therapy, inotropes, and blood products infusion

Palliative care

Warming the infant and making it as comfortable as possible

Advisory committee
This committee should decide on management of PPIs in the healthcare center (hospital) as soon as possible. The committee members: a pediatrician (preferably neonatologist), a gynecologist/ perinatologist, a hospital manager (i.e., chief, deputy), a hospital ethics committee member, and a clergyman.

Maternal/neonatal healthcare facilities
Levels of perinatal care, facilities, and services should be set according to the pregnancy/neonatal risk, which can evaluate from early gestation. The pregnant woman can be admitted to the appropriate hospital according to her pregnancy risk level. There are three levels:

1- Level one: medical facilities available for low-risk mothers/neonates

2- Level two: medical facilities available for moderate-risk mothers/neonates

3- Level three: medical facilities available for high-risk mothers/neonates

[1] The Institute in QOM City. Available from: http://mozooshenasi.ir. (In Persian).

\section{Results}

In the present study, 120 articles, protocols, and guidelines were considered. In addition, 15 sessions were held with 42 active participants, comprising about 588 person-times.

Based on the researchers' opinions and clinical recommendations, the PPIs were divided into four groups; 1) GA below 25 weeks, 2) GA between 25 and 32 weeks, 3) severe asphyxia, and 4) severe life-threatening congenital abnormalities. For the first group, GA up to 24/6 weeks infants, palliative care should be provided in all hospitals (Table 2). Newborns with GA of 25-32 weeks, second category, should be

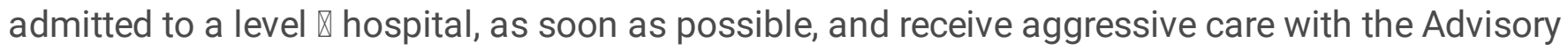

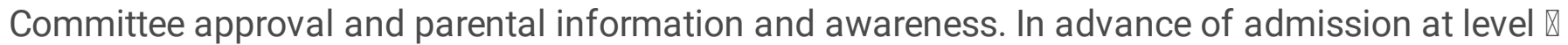
hospitals, these infants should be received supportive care at the level $₫$ and $\otimes$ hospitals (Table 2). 
According to the current guideline for severe asphyxia, palliative care and/or supportive care should be

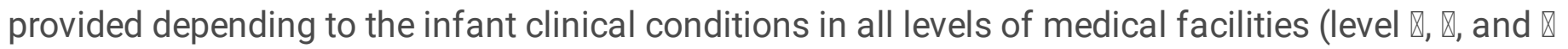
hospitals). In other words, in level I hospital palliative care and in level II and III supportive care should be offered, without referring to other hospitals. In level III educational hospitals, additionally, aggressive care can be applied to infants upon parents' agreement and the ethical committee approval. Diagnosing of severe asphyxia and appropriate clinical care should be approved by two pediatricians (preferably neonatal subspecialists) with the Advisory Committee approval and parental information and awareness. For management these infants, the guideline strongly advices to employee neurologist as much as possible (Table 3).

For the last category of PPIs, infants with severe life-threatening disorders (please see the list of these abnormalities in the <link rid="Sec10">appendix</link>), palliative care should be done with the Advisory Committee approval and parental information and awareness in all hospital levels (Table 4).

The results protocol is presented in Tables 2-4.

Table 2

Clinical recommendations for premature infants (according to gestational age)

\begin{tabular}{|c|c|c|}
\hline \multirow{2}{*}{$\begin{array}{l}\text { Challenge } \\
\text { (condition) }\end{array}$} & \multicolumn{2}{|c|}{ Recommended } \\
\hline & $\begin{array}{l}\text { Level of } \\
\text { mother- } \\
\text { infant } \\
\text { service }\end{array}$ & Care explanations * \\
\hline $\begin{array}{l}\text { Gestational age }<25 \\
\text { week (means } 24 \\
\text { weeks and } 6 \text { days or } \\
\text { less) }\end{array}$ & $\begin{array}{l}\text { All } \\
\text { levels }\end{array}$ & $\begin{array}{l}\text { Apply palliative care (approved by the hospital advisory } \\
\text { Committee and informed parents) }\end{array}$ \\
\hline \multirow[t]{4}{*}{$\begin{array}{l}\text { Gestational age } 25 \\
\text { to } 31 \text { weeks and } 6 \\
\text { days }\end{array}$} & 1 & $\begin{array}{l}\text { Take immediate action to refer to level III. Until transfer, infant } \\
\text { should receive supportive care as explained in Table } 1 \text { (approved } \\
\text { by the hospital advisory committee and informed parents) }\end{array}$ \\
\hline & 2 & Take immediate action to refer to level III. Until transfer, \\
\hline & & $\begin{array}{l}\text { Infant should receive supportive care as explained in the Table } 1 \\
\text { (approved by the hospital advisory committee and informed } \\
\text { parents) }\end{array}$ \\
\hline & 3 & $\begin{array}{l}\text { Apply aggressive care as explained in Table } 1 \text { (approved by the } \\
\text { hospital advisory committee and informed parents) }\end{array}$ \\
\hline
\end{tabular}


Table 3

Clinical recommendation for infants with severe asphyxia

\begin{tabular}{|c|c|c|}
\hline \multirow{2}{*}{$\begin{array}{l}\text { Challenge } \\
\text { (condition) }\end{array}$} & \multicolumn{2}{|c|}{ Recommended } \\
\hline & $\begin{array}{l}\text { Level } \\
\text { of } \\
\text { mother- } \\
\text { infant } \\
\text { service }\end{array}$ & Care explanations * \\
\hline \multirow[t]{3}{*}{$\begin{array}{l}\text { Infant with } \\
\text { severe } \\
\text { asphyxia }\end{array}$} & 1 & $\begin{array}{l}\text { Palliative care and/or supportive care should be provided according to the } \\
\text { infant's clinical condition (needs approval of the hospital advisory } \\
\text { committee and informed parents). Apply appropriate clinical care without } \\
\text { referring to other levels }\end{array}$ \\
\hline & 2 & $\begin{array}{l}\text { Palliative care and/or supportive care should be provided according to the } \\
\text { infant's clinical condition (needs approval of the hospital advisory } \\
\text { committee and informed parents). Apply appropriate clinical care without } \\
\text { referring to other levels }\end{array}$ \\
\hline & 3 & $\begin{array}{l}\text { Palliative care and/or supportive care should be provided according to the } \\
\text { infant's clinical condition (needs approval of the hospital advisory } \\
\text { committee and informed parents) }\end{array}$ \\
\hline
\end{tabular}

Table 4

Clinical recommendations for infants with inherited metabolic disorders or severe life-threatening congenital abnormalities

\begin{tabular}{|c|c|c|}
\hline \multirow{2}{*}{$\begin{array}{l}\text { Challenge } \\
\text { (condition) }\end{array}$} & \multicolumn{2}{|c|}{ Recommended } \\
\hline & $\begin{array}{l}\text { Level of } \\
\text { mother- } \\
\text { infant } \\
\text { service }\end{array}$ & Care explanations * \\
\hline \multirow{3}{*}{$\begin{array}{l}\text { Infant with } \\
\text { severe life- } \\
\text { threatening } \\
\text { disorders }\end{array}$} & 1 & $\begin{array}{l}\text { Palliative care should be provided according to the infant's clinical } \\
\text { condition (needs approval of the hospital advisory committee and } \\
\text { informed parents). Apply clinical care without referring to other levels }\end{array}$ \\
\hline & 2 & $\begin{array}{l}\text { Palliative care should be provided according to the infant's clinical } \\
\text { condition (needs approval of the hospital advisory committee and } \\
\text { informed parents). Apply clinical care without referring to other levels }\end{array}$ \\
\hline & 3 & $\begin{array}{l}\text { Palliative care should be provided according to the infant's clinical } \\
\text { condition (needs approval of the hospital advisory committee and } \\
\text { informed parents). }\end{array}$ \\
\hline
\end{tabular}

In addition, using to the experts' opinion, "neonatal moribund" in the clinical setting was defined as follows: 
1. Infants born before 25 weeks' gestation (up to 24 weeks and 6 days)

2. Confirmed cases of severe asphyxia according to Table 1 confirmed by of two pediatricians (preferably neonatal subspecialists). Consultation with a pediatric neurologist is recommended.

3. Confirmed cases of severe lethal malformations and/or severe lethal chromosomal/ genetic disorders (<link rid="Sec10">Appendix</link $>$ )

This definition was very helpful for clarifying the issue for religious authorities and obtaining the jurisprudential ruling (fatwa).

\section{Discussion}

This study was the first nationwide attempt to prepare a comprehensive framework for professional management of a challenging medical situation and to gain insight into the problems of PPIs, such as extremely preterm cases as well as infants with severe asphyxia and lethal congenital anomalies/severe inborn errors of metabolism. This guideline can support prenatal counseling in Iranian hospitals with various medical facilities (i.e., level I/II/III neonatal care) to achieve more homogeneity in newborn medical care.

There are several considerations in the literature and national guidelines with different approaches to problems of PPI. For instance, in Taiwan, $86 \%$ of the NICU nurses agreed with "do-not-resuscitate" and $70 \%$ agreed with "withdrawal of advanced treatment" for severely ill neonates in the NICU [16]. However, Huang et al. (2013) found that $70 \%$ of the practitioners did not support "withdrawal of life-preserving treatment", mainly due to religious beliefs. Additionally, many other factors can influence the healthcare practitioners' decision, such as the family size (number of children), workload on the hospital personnel, the parents' preference, infant's sex, hospital facilities or resources, history of infertility, and socioeconomic status of the family $[17,18]$. Thus, according to the German Medical Association, the final decisions should be made regarding different situations and cannot be the same for all PPIs [19].

Prenatal counseling for PPIs' families is a difficult task for medical professionals. In most cases, the parents themselves may not wish to be the primary decision makers in withdrawing care. We suggest that parents should be informed about almost all medical procedures.

There are two main approaches to PPIs: 1) priority to save infant's life until death is inevitable, 2) waiving advanced medical care when very low quality of life is prospected [20]. These two approaches have own supporters/opponents. The present study was conducted to introduce a new protocol based on Shiite (Shiah) jurisprudence considerations in which all medical practitioners are obliged to preserve human life in any possible way until inevitable death is predicted in a short period of time (in a few days). This definition is equivalent to "dying" and "life-limiting conditions" in a study by Nice, which states that palliative care should be provided until "death in peace" occurs [21]. However, the Nice's guidelines did not mention any clear criteria for decision-making by involving the healthcare team while our guideline is more transparent with measurable criteria to make a diagnosis (i.e., for moribund), which can be applied in all parts of Iran without individual interpretation (Table 1 and 5). 


\section{Extremely premature infants}

Compared to other types of PPIs, prematurity has a clear cut-off point (GA) for classification upon which the majority of the studies in the literatures and guidelines have focused [4]. Along with the increase in the survival rate of extremely premature infants in recent years, the risk of morbidities and a "low quality of life" has increased as well. According to GA, the infants were divided to two groups: newborns with GA below 25 weeks and those with GA between 25 and 32 weeks. For the first group, the present guideline recommends palliative care in all levels of mother-infant medical services. Supportive care should be launched immediately for the infants born between 25 and 32 weeks' gestation. Then, the newborn should be transfer to a level III hospital as soon as possible.

Several recent studies (between 2000 and 2020) have been conducted in NICUs of different areas of Iran. The results have not shown favorable outcomes in infants born before 25 weeks' GA. For infants born between 26 and 28 weeks' gestation, studies have shown an infant mortality rate of $48-70 \%$ in Iran [10, 22-25]. Similarly, a meta-analysis by Sahebi et al. (2018) using the Middle East population data revealed a $32 \%$ infant mortality rate for VLBW infants ( $\leq 30$ weeks), while the rate was higher in African countries (55\%) [15]. Regardless of IUGR infants, this is rate of outcomes for newborns born before 30 weeks of gestation.

The results of the present qualitative study suggest that there are not enough facilities for swift transfer of all very premature babies to NICUs in many Iranian cities. According to panel discussions, most of the province capitals have NICUs. However, other facilities and hospital staff skills may be insufficient to take care of babies born before 25 weeks' gestation. Many guidelines are based on the assumption that very premature infants are only born at level III medical centers. However, the present study provided a guideline for medical teams of level I and II hospitals as well to manage such infants.

The Switzerland guideline (2017) recommends palliative care for newborns with a gestational age of 22 $0 / 7$ to 23 6/7 weeks without indications for Cesarean section and aggressive care, even when parents disagree with it. They recommend Cesarean section and aggressive care for all newborns with a GA of 25 $0 / 7$ to $256 / 7$ weeks without parents' consultation. The GA between these two groups is defined as the 'gray zone', which has some criteria for making treatment decisions [26].

Although our guideline and the Switzerland guideline are similar for birth at 250/7 weeks of gestation and above, they are different for lower gestational ages ( $<25$ weeks) due to differences in medical resources and country territorial area.

The Fetus and Newborn Committee of the Canadian Pediatric Society has almost the same recommendations for infants born before or at 21 (palliative care) and between 22 0/7-25 6/7 weeks' gestation (gray zone). In addition, the Canadian recommendation is similar to our guideline for a gestational age of $240 / 6$ weeks. The Canadian protocol also has a gray zone with other factors in addition to GA for decision-making [27]. By improving medical facilities in the country, this guideline can be modified in the future. 
Similarly, Hussein et al. (2010) recommended that taking care of newborns with GA $\leq 28$ or birth weight of $\leq 1000 \mathrm{~g}$ may not be a good choice in low-income countries because of limitations in medical resources and high mortality rates among them. Instead, healthcare professional should focus on the newborns at higher GA [28]. In addition, recent Australian guidelines suggest that clinician should primarily make decisions for infants born at 25 weeks' gestation [29]. However, the healthcare providers believe that parents prefer to provide medical care for infants born at or after 23 weeks' gestation [30].

According to the American Academy of Pediatrics and the American Heart Association, resuscitation of newborns with a GA of $<23$ weeks has no indication due to the high risk of mortality and/or unacceptable consequences in the surviving infants [31]. On the other hand, the Dutch guideline on perinatal practice in extremely premature delivery lowers the limit offering intensive care from $240 / 7$ weeks to $256 / 7$ weeks' gestation [32]. The guideline does recommend transfer to a tertiary center at $23+4 / 7$ weeks to allow sufficient time for (repeated) counseling within $24 \mathrm{~h}$ in the tertiary center [30].

\section{Infants with severe asphyxia}

The present guideline recommends supportive/ palliative care for severe asphyxia according to the infant's clinical condition, upon approval of the hospital advisory committee and informed parents, without referring to a hospital with higher levels of care.

Since diagnosing asphyxia and its severity has always been a challenging situation for neonatologists and perinatologists, we defined severe asphyxia as a condicition in which no treatment can cure the asphyxiated newborn and only postpones death. This definition is similar to "very poor prognosis" in the most of other studies [33-36]. Furthermore, in the present study, a diagnosis of severe asphyxia must be confirmed by at least two pediatricians or neonatal specialists, and neurological consultant can be helpful. In a study by Bhata et al (2006), palliative care was suggested for three groups of newborns: those born before 23 weeks' gestation, those born with fatal congenital abnormalities, and those not responding to aggressive therapy (inevitable death) [37]. Similarly, we recommend palliative care for severe asphyxia whenever the newborn does not respond to aggressive treatment. Since it is not possible to diagnose severe asphyxia at the time of delivery, the medical team must perform resuscitation and necessary treatments using medical facilities. Whenever no response to treatment is observed and the newborn's condition meets the severe asphyxia criteria, only palliative care is indicated. However, only a few medical centers have the facilities for providing hypothermic care for HIE infants in Iran.

\section{Infants with severe life-threatening congenital abnormalities}

The present framework suggests palliative care, according to the infants' clinical condition, for severe life threatening genetic/congenital abnormalities and/or errors of metabolism without referring to hospitals offering higher levels of medical care. The clinical support should be done after obtaining approval from the hospital advisory committee and informed parent. According to the German Medical Association, decisions on the optimal care regarding these critically ill neonates are dependent on the situation and cannot be generalized to all cases [19]. A survey in Iran reported that the majority of the NICU 
pediatricians and neonatologists did not agree to aggressive care in infants with severe anomalies including genetic disorders and advanced asphyxia [38].

There is not a constant definition/criterion for decision making about the ethical aspects of lethal congenital anomalies in the prenatal setting [39]. Wilkinson separated these newborns into two groups: "fetal neonatal death" in which, despite treatment, all newborns would be expired without exception, and "usual fetal neonatal death" in which, despite treatment, newborn death is often inevitable. Anencephaly, trisomy 13 and 18, Potter syndrome, holoprosencephaly, and thanatophoric dwarfism (thanatophoric dysplasia) have been considered as the most common fatal malformations. However, an important question is as infants with this type of disorder live for weeks to one year, should it be considered a fatal condition. Finally, palliative care would be ethically acceptable if a very high probability of early death or severe disability is predicted after adequate aggressive treatment is provided [39]. We suggested palliative care for congenital fatal malformations (Appendix). In addition, a study in Saudi Arabia suggested palliative care according to Islamic ethics code for severe fatal malformations with a prospective survival time between one hour and 30 days [40], with a larger list than the list suggested by Wilkinson study and shorter list compared to our guideline [39].

According to the Switzerland guidelines, if the healthcare resources are limited and effective treatment needs to be prioritized, the care should be given to cases who may have more marginal benefits in receiving it. It is not moral to deprive only a certain group of very poor prognosis patients (i.e., very premature newborns) from receiving aggressive care [26]. Thus, our guideline complies with this approach for these fatal anomalies. In other words, because almost all cases with a poor/very poor prognosis are included in our guideline (extreme premature infants, severe asphyxia, severe lifethreatening congenital abnormalities), the present guideline complies with the Switzerland guideline.

Although there is no significant difference in the management of two groups of PPIs (asphyxia and severe fatal abnormalities) in this study, the guidelines can be revised at any time considering the very quick advance in medical knowledge, skills, and equipment at level III neonatal care centers. For example, before 2000, cardiac surgery for transposition of great arteries (TGA) or congenital diaphragmatic hernia $(\mathrm{CDH})$ was associated with a little chance of success in our country. However, in the recent years, infants with these anomalies are not at high risk of death [41-47]. Another important recommendation of the present guideline is that the medical treatment should be allowed to offer aggressive care to some infants receiving palliative care to 1 ) investigate and evaluate new and/or aggressive treatment methods, 2) increase the staff's experience and capabilities, and 3) train subspeciality residents. In this regard, the medical team should provide a clear and sufficient explanation to the parents about treatment strategy change (from palliative care to aggressive care), obtain their consent, and consider all ethical issues (like obtaining ethical clearance from the ethics committee). In such cases, medical ethics should be followed, the parents should receive psychological support, and all procedures should be carried out free of charge.

\section{Suggestions}


We suggest complementary studies within five years of implementing this guideline to assess its impacts and revise its recommendations. For example, it may be necessary to revise the "gray zone" recommendations by including other factors in PPI survival and/or changes in the list of abnormalities.

\section{Conclusion}

A guideline was developed in the present study in collaboration with Islamic religious leaders, specialist/subspecialist physicians, healthcare professionals, medical ethicists, and parents. In addition, we reviewed the published papers and guidelines about PPI managements and its changes against the background of accumulated evidence drawn from different countries during the past few years. Thus, the present guideline covers the main areas of concern and clearly states the responsibilities for decisionmaking regarding prenatal counseling for PPIs across Iran. Finally, the present guideline was approved by Iranian Supreme Ethics Council and available in their website at the following link https://medethicsnet.behdasht.gov.ir.

\section{Abbreviations}

PPIs: Poor prognosis infants; ELBW: Extremely low birth weight; WHO: World health organization; IEM: Inborn errors of metabolism; HIE: Hypoxic-ischemic encephalopathy; NICUs: Neonatal intensive care units; TGA: Transposition of great arteries; $\mathrm{CDH}$ : Congenital diaphragmatic hernia; LoV: Limit of viability; TUMS: Tehran University of Medical Sciences; MHME: Ministry of Health and Medical Education; NCEC: National Clinical Ethics Committee.

\section{Declarations}

\section{Acknowledgements}

We would like to thank Dr. Alireza Marandi, President of Medical Sciences Academy, Iran; Dr. Ghasem Janbabai, Deputy for Treatment, MHME, Iran; Faranak Nadarkhani, President of Standardization of Guidelines Office, MHME, Iran; Mohammad Hossein Fallahzadeh, President of the Institute of Theology of Jurisprudential Rulings, and other members of the institute; and a special thanks to members of NCEC and Supreme Council on Medical Ethics, for kindly collaboration with the current study. The authors would like to thank Ameneh Abruon and Sayeh Mirzatavoli for the nurses' interview arrangement; Dr. Yasamin Mohammadzadeh for the panels discussion arrangement; Zohre Janani for the parent's interview arrangement; all the hospitals' medical staffs, and mother/infant pairs for their cooperation and assistance.

\section{Authors' contributions}

Design of the study: FSN, MS

Managing interviews with the PPIs parents and the hospitals' nurses: MS, NF 
Panel discussion with the specialists: FSN, MS, FA, AP, ESG

Panel discussion with the religious masters (Shia clerics): MMA, FSN

Codification the guideline: FSN, MK

Discussion the findings and manuscript writing: FSN, MS, NF, MV

All authors read and approved the final manuscript.

\section{Funding}

The present study has been financially supported in two phases: phase one by TUMS (\#27833), Tehran, Iran, and phase two by the Guideline Standardization Office (\#39599), MHME, Iran.

\section{Availability of data and materials}

The study data is available from the corresponding author upon request.

\section{Ethics approval and consent to participate}

This study protocol was reviewed and approved by scientific committee of Maternal, Neonatal, and Fetal Research Center, TUMS, Tehran, Iran. Then the study protocol was ethically approved by the Research Ethics Committee, Tehran University of Medical Sciences (IR.TUMS.VCR.REC.1397.658). All the study was carried out in accordance with national codes of ethics for research on human subjects which are compatible with international research ethics standards included in the Declaration of Helsinki. After receiving approval, the survey was conducted under the supervision of Maternal, Neonatal, and Fetal Research Center, TUMS, Tehran, Iran. All participants were invited for participation in this study and informed consent was obtained from all participants.

\section{Consent for publication}

All the authors have an agreement to publish the paper in the present context and format.

\section{Competing interests}

The authors have no conflicts of interest to disclose.

\section{Author details}

${ }^{1}$ Maternal, Fetal and Neonatal Health Research Center, Institute of Family Health, Tehran University of Medical Sciences, Tehran, Iran. ${ }^{2}$ Medical Ethics and History of Medicine Research Center, Tehran University of Medical Sciences, Tehran, Iran. ${ }^{3}$ Islamic Maaref Department, Tehran University of Medical Sciences, Tehran, Iran. ${ }^{4}$ Department of Epidemiology and Environmental Health, Faculty of Medicine, Juntendo University, Tokyo, Japan. ${ }^{5}$ Treatment Affairs, Ministry of Health and Medical Education, Tehran, 
Iran. ${ }^{6}$ Medical Ethics and History of Medicine Research Center, Tehran University of Medical Sciences, Tehran, Iran. ${ }^{7}$ Department of Medical Ethics, Faculty of Medicine, Tehran University of Medical Sciences, Tehran, Iran. ${ }^{8}$ Medical Ethics and History of Medicine Research Center, Faculty of Medicine, Tehran University of Medical Sciences, Tehran, Iran. ${ }^{9}$ Breast Feeding Research Center, Institute of Family Health, Tehran University of Medical Sciences, Tehran, Iran.

\section{References}

1. World Health Organization. WHO Child Growth Standards Backgrounder 2.2018. https://www.who.int/childgrowth/2_why.pdf.

2. Martin RJ, Fanaroff AA, Walsh M. Diseases of the Fetus and Infant in Fanaroff And Martin's Neonatal-Perinatal Medicine. 10th ed. Philadelphia: Elsevier/Mosby; 2015. p. 924.

3. Martin RJ, Fanaroff AA, Walsh M. Fanaroff and Martin's Neonatal-Perinatal Medicine - Diseases of the Fetus and Infant. 10th ed. Seminars in Fetal and Neonatal Medicine. Philadelphia: Elsevier/Mosby; 2015. p. 904.

4. Shariat M, Asghari F, Motevasel Arani M, Farrokhzad N, Mohammadzadeh Y, Mohammadi Shahboulaghi $F$, et al. Guideline developing for determination of limit of providing medical care in poor prognosis neonates [Internet]. https://research.tums.ac.ir/print_research_new.phtml?

5. Martin RJ, Fanaroff AA, Walsh M. Fanaroff and Martin's Neonatal-Perinatal Medicine - Diseases of the Fetus and Infant. In: Laventhal NT, Fanaroff JM, editors. Medical Ethics in Neonatal Care. 11th ed. Philadelphia: Elsevier/Mosby; 2020. p. 173-245.

6. Seri I, Evans J. Limits of viability: definition of the gray zone. J Perinatol. 2008;28(S1):S4-8. https://doi.org/10.1038/jp.2008.42.

7. Morgan MA, Goldenberg RL, Schulkin J. Obstetrician-gynecologists' practices regarding preterm birth at the limit of viability. J Matern Neonatal Med. 2008;21(2):115-21. https://doi.org/10.1080/14767050701866971.

8. Halamek LP. Prenatal Consultation at the Limits of Viability. Neoreviews. 2003;4(6):153e - 156. https://doi.org/10.1542/neo.4-6-e153.

9. Su B-H, Hsieh W-S, Hsu C-H, Chang J-H, Lien R, Lin C-H, et al. Neonatal Outcomes of Extremely Preterm Infants from Taiwan: Comparison with Canada, Japan, and the USA. Pediatr Neonatol. 2015;56(1):46-52. https://doi.org/10.1016/j.pedneo.2014.05.002.

10. Dalili H, Fallahi M, Moradi S, Nayeri F, Shariat M, Rashidian A. Clinical outcome and cost of treatment and care for neonates less than 1000 grams admitted to Vali-e ASR Hospital. Heal Econ Rev. 2014;4(1):1-6. https://doi.org/10.1186/s13561-014-0021-7.

11. Kaempf JW, Tomlinson MW, Campbell B, Ferguson L, Stewart VT. Counseling Pregnant Women Who May Deliver Extremely Premature Infants: Medical Care Guidelines, Family Choices, and Neonatal Outcomes. Pediatrics. 2009;123(6):1509-15. https://doi.org/10.1542/peds.2008-2215. 
12. Berger TM, Bernet V, El Alama S, Fauchère J-C, Hösli I, Irion O, et al. Perinatal care at the limit of viability between 22 and 26 completed weeks of gestation in Switzerland. 2011 revision of the Swiss recommendations. Swiss Med Wkly. 2011;141:w13280. https://doi.org/10.4414/smw.2011.13280.

13. Pignotti MS, Donzelli G. Perinatal care at the threshold of viability: An international comparison of practical guidelines for the treatment of extremely preterm births. Pediatrics. 2008 Jan;121(1):e1938. https://doi.org/10.1542/peds.2007-0513.

14. Berger TM, Büttiker V, Fauchère JC, Holzgreve W, Kind C, Largo R, et al. Empfehlungen zur Betreuung von / Frühgeborenen an der Grenze der Lebensfähigkeit / (Gestationsalter 22...26 SSW). Schweizerische Ärztezeitung. 2002;83:1589-95. https://doi.org/10.4414/saez.2002.09137.

15. Nayeri F, Emami Z, Mohammadzadeh Y, Shariat M, Sagheb S, Sahebi L. Mortality and Morbidity Patterns of Very Low Birth Weight Newborns in Eastern Mediterranean Region: A Meta-Analysis Study. J Pediatr Rev. 2018;7(2):67-76. https://doi.org/10.32598/jpr.7.2.67.

16. Ghaffari Saravi V, Zarghami M, Sheikh Moonesi F, Moodi S, Khadem F, Karami M. Attitude and practice of pediatrics and gynecology physicians in the cities of Sari and Babol regarding noninitiation and discontinuation of resuscitation in cases of gravely ill newborns. J Maz Univ Med Sci. 2009;19(70):33-40.

17. Huang LC, Chen CH, Liu HL, Lee HY, Peng NH, Wang TM, et al. The attitudes of neonatal professionals towards end-of-life decision-making for dying infants in Taiwan. J Med Ethics. 2013;39(6):382-6. https://doi.org/10.1136/medethics-2011-100428.

18. Banazadeh M, Khanjari S, Naddaf A, Oskouie F. Healthcare professionals-related factors affecting parents' participation in decision making for neonates with life-threatening conditions: A qualitative study. J Eval Clin Pract. 2021;27(4):885-897. https://doi.org/10.1111/jep.13492.

19. Willems DL, Verhagen AAE, van Wijlick E, Committee End-of-Life Decisions in Severely III Newborns of Royal Dutch Medical Association. Infants' Best Interests in End-of-life Care for Newborns. Pediatrics. 2014;134(4):e1163-8. https://doi.org/10.1542/peds.2014-0780.

20. Rebagliato M, Cuttini M, Broggin L, Berbik I, de Vonderweid U, Hansen G, et al. Neonatal end-of-life decision making: Physicians' attitudes and relationship with self-reported practices in 10 European countries. JAMA. 2000;284(19):2451-9. https://doi.org/10.1001/jama.284.19.2451.

21. NICE. Recommendations | End of life care for infants, children and young people with life-limiting conditions: planning and management | Guidance | NICE. 2019. https://www.nice.org.uk/guidance/ng61/chapter/recommendations\#approaching-the-end-of-life. Accessed 25 July 2019.

22. Rezaeizadeh G, Dalili H, Shariat M, Fallahi M, Other Members Of Maternal Group O, Nayeri F. The Iranian Neonatal Registry: Primary Results. Arch Iran Med. 2018;21(4):145-52.

23. Saleh Gargari S, Kashanian M, Zendedel H, Nayeri F, Shariat M, Haghollahi F. Survival and Risk Factors of Extremely Preterm Babies (< 28 weeks) in the Three Iranian Hospitals Soraya. Acta Medica Iranica. 2018;56(3):181-8. 
24. Nayeri F, Dalili H, Nili F, Amini E, Ardehali A, Khoshkrood Mansoori B, et al. Risk factors for neonatal mortality among very low birth weight neonates. Acta Med Iran 2013;51(5):297-302.

25. Nayeri F, Amini E, Shariat M, Mansoori B. Life expectancies and outcomes in extremely low birth weight neonates - Tehran University Medical Journal TUMS Publications. Tehran Univ Med J. 2008;66(4):288-93.

26. Fischer N, Steurer MA, Adams M, Berger TM. Survival rates of extremely preterm infants (gestational age <26 weeks) in Switzerland: Impact of the Swiss guidelines for the care of infants born at the limit of viability. Arch Dis Child Fetal Neonatal Ed. 2009;94(6):F407-13. http://doi.org/10.1136/adc.2008.154567.

27. Lemyre B, Moore G, Canadian Paediatric Society, Fetus and Newborn Committee. Counselling and management for anticipated extremely preterm birth | Canadian Paediatric Society. Paediatr Child Heal. 2017;22(6):334-41. http://doi.org/10.1093/pch/pxx058.

28. Parappil H, Rahman S, Salama H, Al Rifai H, Parambil NK, Ansari W El. Outcomes of 28+1 to 32+0 weeks gestation babies in the State of Qatar: Finding facility-based cost effective options for improving the survival of preterm neonates in low income countries. Int J Environ Res Public Health. 2010;7(6):2526-42. http://doi.org/10.3390/ijerph7062526.

29. Ireland S, Ray R, Larkins S, Woodward L. Factors influencing the care provided for periviable babies in Australia: A narrative review. Reprod Health. 2015;12:108. http://doi.org/10.1186/s12978-0150094-8.

30. Gallagher K, Martin J, Keller M, Marlow N. European variation in decision-making and parental involvement during preterm birth Arch Dis Child Fetal Neonatal Ed. 2014;99(3):F245-9. http://doi.org/10.1136/archdischild-2013-305191.

31. Weiner GM, Zaichkin J, Kattwinkel, editors. Textbook of Neonatal Resuscitation (NRP). 7th Ed. Am Acad Pediatrics, 2016.

32. Geurtzen MR, Van Heijst A, Hermens R, Scheepers H, Woiski M, Draaisma J, et al. Preferred prenatal counselling at the limits of viability: A survey among Dutch perinatal professionals. BMC Pregnancy Childbirth 2018;18(1):7. http://doi.org/10.1186/s12884-017-1644-6.

33. Martin RJ, Fanaroff AA, Walsh M. Diseases of the Fetus and Infant in Fanaroff And Martin's Neonatal-Perinatal Medicine. 11th ed. Philadelphia: Elsevier/Mosby; 2020. p. 3127-3204.

34. Kim YE, Sung SI, Chang YS, Ahn SY, Sung DK, Park WS. Thrombin preconditioning enhances therapeutic efficacy of human wharton's jelly-derived mesenchymal stem cells in severe neonatal hypoxic ischemic encephalopathy. Int J Mol Sci. 2019;20(10): 2477. http://doi.org/10.3390/ijms20102477.

35. Azzopardi D V., Strohm B, Edwards AD, Dyet L, Halliday HL, Juszczak E, et al. Moderate Hypothermia to Treat Perinatal Asphyxial Encephalopathy. N Engl J Med. 2009;361(14):1349-58. Available from: http://doi.org/10.1056/NEJMoa0900854.

36. Shankaran S, Laptook AR, Ehrenkranz RA, Tyson JE, McDonald SA, Donovan EF, et al. Whole-Body Hypothermia for Neonates with Hypoxic-Ischemic Encephalopathy. N Engl J Med. 
2005;353(15):1574-84. http://doi.org/10.1056/NEJMcps050929.

37. Bhatia J. Palliative care in the fetus and newborn. J Perinatol. 2006;26(1):S24-6. http://doi.org/10.1038/sj.jp.7211468.

38. Nayeri F, Asghari F, Baser A, Janani L, Shariat M, Eabrhim B. Views and decisions of physicians in encountering neonates with poor prognosis. Arch Iran Med. 2017;20(3):172.

39. Wilkinson $D$, Crespigny $L$ de, Xafis $V$. Ethical language and decision-making for prenatally diagnosed lethal malformations. Semin Fetal Neonatal Med. 2014; 19(5):306-11. http://doi.org/10.1016/j.siny.2014.08.007.

40. Abdelbasit O, Seidahmed M, Alhussein K, Miqdad A, Mohsen A, Khalil M, et al. The ethics of lethal congenital malformations. Is palliative therapy possible? J Clin Neonatol. 2019;8(4):216. http://doi.org/10.4103/jcn.JCN_39_19.

41. Mirzaaghayan MR, Ghamari A, Amini S, Rabbani A, Mohebbi A, Moghadam EA. A 3-year single surgeon experience of the arterial switch operation. Iran J Pediatr. 2020;30(5):1-6. http://doi.org/10.5812/ijp.98540.

42. Shahmohammadi A, Mortezaian H, Alipour MR. One-year outcome of Senning operation in children with transposition of the great vessels. Tehran Univ Med J TUMS Publ. 2011;69(5):289-95.

43. Hiradfar M, Shojaeian R, Joodi M, Nazarizadeh R, Gharavi M, Sabzevari A. Thoracoscopic Congenital Diaphragmatic Hernia repair in neonate: The First Experience of Iranian Group. Iran J Pediatr Surg. 2015;1(2):66-70. https://doi.org/10.22037/irjps.v1i2.11529.

44. Musavi M, Rafeie MH, Hosseinpour M, Ahmadi $\mathrm{B}$. The predicting values of Pao2/Fio2 ratio in survival of neonates with Congenital Diaphragmatic Hernia. Iran J Pediatr Surg. 2016;2(2):66-70. https://doi.org/10.22037/irjps.v2i2.14404.

45. Ghavami-Adel M, Dalili H, Shariat M, Ghorbansabagh V, Nayeri F, Shirvany TE, et al. Factors Affecting Survival in Congenital Diaphragmatic Hernia: A Prospective study at a Tertiary Center. J Neonatal Surg. 2015; 8(4):30. https://doi.org/10.47338/jns.v8.355.

46. Dehdashtian M, Bashirnejad S, Malekian A, Aramesh MR, Aletayeb MH. Seasonality, Epidemiology and Outcome of Congenital Diaphragmatic Hernia in South West of Iran. J Neonatal Surg. 2017;6(2):28. https://doi.org/10.21699/jns.v6i2.506.

47. Baghaei R. Long-term coronary artery outcome after arterial switch operation for transposition of the great arteries. Iranian Journal of Cardiac Surgery. 2011;3(1):54.

\section{Supplementary Files}

This is a list of supplementary files associated with this preprint. Click to download.

- Appendix.docx 